\title{
Outflow Graft Foreign Body Reaction and Thrombosis in HeartMate III Left Ventricular Assist Device
}

\author{
Paulino Alvarez ${ }^{1}$, Dennis Firchau ${ }^{1}$, Robert Humble ${ }^{1}$, and Alexandros Briasoulis ${ }^{1}$ \\ ${ }^{1}$ University of Iowa
}

February 25, 2021

\begin{abstract}
Outflow cannula occlusion is an infrequent complication occurring among recipients of continuous flow left ventricular assist devices (LVAD). Hereby, we present a case of intrinsic and extrinsic outflow cannula obstruction resulting in cardiogenic shock and multiorgan failure.

\section{Outflow Graft Foreign Body Reaction and Thrombosis in HeartMate III Left Ventricular Assist} Device

Paulino Alvarez MD ${ }^{a}$, Robert Humble MD ${ }^{\mathrm{b}}$, Dennis Firchau MD ${ }^{\mathrm{b}}$, Alexandros Briasoulis $\mathrm{MD} \mathrm{PhD}^{\text {a }}$

${ }^{a}$ Department of Cardiovascular Medicine, University of Iowa Hospitals and Clinics, Section of Heart Failure and Transplantation

${ }^{\mathrm{b}}$ Department of Pathology, University of Iowa Hospitals and Clinics,
\end{abstract}

Key words : outflow cannula, LVAD, thrombosis

Running title : outflow cannula obstruction in LVAD

Word count : 758

Disclosures : None, Funding : None

Address of correspondence:

Alexandros Briasoulis

Section of Heart Failure and Transplant, Division of Cardiovascular Diseases

University of Iowa Hospitals and Clinics,

200 Hawkins Dr, Iowa City, IA 52242

Office: $319-678-8418$

Fax: $319-353-6343$

E-mail: alexbriasoulis@gmail.com

Abstract: Outflow cannula occlusion is an infrequent complication occurring among recipients of continuous flow left ventricular assist devices (LVAD). Hereby, we present a case of intrinsic and extrinsic outflow cannula obstruction resulting in cardiogenic shock and multiorgan failure. 


\section{Data availability : upon request by Editors}

International Review Board approval : Waived, as this is a single case report

Informed consent : This is a case report, informed consent was not obtained as the patient did not survival to hospital discharge.

Case Description: A 66 year old Caucasian male with medical history of emphysema, ischemic cardiomyopathy and stage D heart failure status post HeartMate 3 LVAD implantation as destination therapy eighteen months before the index admission for fever and shortness of breath. His international normalized ratio was in therapeutic range between 2 and 3 on admission. Despite early symptomatic improvement of the respiratory symptoms with intravenous diuresis and antibiotics on the fifth day of this hospital stay he developed low flow alarms, decreased power but stable LVAD speed (5600 rpm) (Figure A). A bedside echo showed persistent aortic valve opening in spite of increasing speed to 6000rpm. Outflow cannula obstruction was suspected and a CT scan of the chest was performed which revealed an irregular circumferential hypodensity in the outflow cannula of the left ventricular assist device consistent with thrombus or extrinsic compression from material between the outflow and the bend relief material, without evidence of acute kinking of the outflow graft (Figures B-D). Of note, a previous CT angiography of abdomen did not reveal evidence of outflow graft occlusion (Figure E). The became became hypotensive and was intubated because of altered mentation and worsening respiratory distress. He was resuscitated with infusion of norepinephrine and dobutamine. The lactate dehydrogenase (LDH) and plasma free hemoglobin were not elevated. The patient was started intravenous anticoagulation with intravenous heparin in anticipation of surgical intervention. However, in the first 48 hours of initiation of intravenous anticoagulation, he developed diffuse alveolar hemorrhage diagnosed with a CT chest and confirmed by bronchoscopy. His overall condition progressed rapidly to multiorgan failure (acute kidney and liver injury). His hemodynamics at the time suggested cardiogenic shock with Fick and thermodiluation cardiac index of $<1.8 \mathrm{l} / \mathrm{minute} / \mathrm{m}^{2}$, mixed venous saturations $<40 \%$, and elevated pulmonary capillary wedge pressure over $20 \mathrm{mmHg}$ and central venous pressure of $14 \mathrm{mmHg}$. After meeting with family of the patient, the decision of the multi-disciplinary team was to proceed with comfort care measures and hospice on the basis of deteriorating multi-organ function, low likelihood of lung function recovery in the context of acute on chronic lung disease, and poor candidacy for cardiac surgery.

Autopsy exam revealed instrinsic and extrinsic outflow graft obstruction. The extrinsic obstruction consisted of fibrinous tissue between the woven polyester tube and the bend relief (Figures F-N).

\section{Discussion}

As shown in analyses of the randomized unblinded trial (Multicenter Study of MagLev Technology in Patients Undergoing Mechanical Circulatory Support Therapy with HeartMate 3 [MOMENTUM 3]), the design of HeartMate 3 LVAD results in enhanced hemocompatibility and lower risk of pump thrombosis and reoperations to replace or remove the pump compared with axial-flow LVADs.(1,2) . However, outflow graft occlusion may lead to acute hemodynamic compromise, hence prompt diagnosis and treatment are imperative to avert poor outcomes. The mechanisms involved in ouflow cannula obstruction include twisting of the outflow graft, intraluminal obstruction or extrinsic compression.(5) Duero Posada et al reported two patients who developed a thrombus between the outflow graft and bend relief that caused extrinsic compression provoking near total occlusion and hemodynamic compromise leading to urgent cardiac transplantation. (6) In our case, the obstruction occurred at the level of the bend relief and consisted predominantly of chronic inflammatory with evidence of foreign body reaction. In an analysis of 26 HVAD outflow grafts tissue ingrowth was present in $24(92 \%)$. The most common site was distal anastomosis with microscopic evidence of chronic inflammatory infiltrates, giant cells, neointima formation and fibrosis. The median depth of tissue was $1 \mathrm{~mm}$ leading to reduced pump flow that didn’t translate into hemodynamic comprise. (7)

Our case highlights the morbidity and mortality associated with outflow cannula occlusion. Albeit infrequent, outflow cannula occlusion should be suspected in LVAD recipients with low flows, normal LDH, and evidence of poor LV unloading and decreased cardiac output. For patients with LVADs as bridge to transplantation, upgrade to higher listing status and hemodynamic support with inotropes or temporary mechanical support 
are viable options. Alternatively, replacement of outflow graft, LVAD exchange or in patients with prohibitive surgical risk, bend relief dissection and liberalization of the proximal outflow cannula through subcostal incision, and percutaneous endovascular stenting have been reported $(9,10)$. The severity of pulmonary complications and multi-organ failure precluded those approaches in our patient.

Recognizing the mechanism of obstruction of the outflow graft is key to dictate appropriate therapeutic strategies and prevent complications. The role of foreign body reaction as a potential contributor of outflow graft obstruction at the level of the bend relief should be confirmed by careful histopathologic analysis of explanted left ventricular assist device systems.

\section{References}

1. Mehra MR, Uriel N, Naka Y et al. A Fully Magnetically Levitated Left Ventricular Assist Device Final Report. N Engl J Med 2019;380:1618-1627.

2. Uriel N, Colombo PC, Cleveland JC et al. Hemocompatibility-Related Outcomes in the MOMENTUM 3 Trial at 6 Months: A Randomized Controlled Study of a Fully Magnetically Levitated Pump in Advanced Heart Failure. Circulation 2017;135:2003-2012.

3. Mehra MR, Salerno C, Naka Y et al. A tale of the twist in the outflow graft: An analysis from the MOMENTUM 3 trial. J Heart Lung Transplant 2018;37:1281-1284.

4. Duero Posada JG, Moayedi Y, Alhussein M et al. Outflow Graft Occlusion of the HeartMate 3 Left Ventricular Assist Device. Circ Heart Fail 2017;10.

5. Jain P, Robson D, Shehab S et al. In vivo tissue reaction within the outflow conduit in patients supported by HeartWare HVAD. Cardiovasc Pathol 2020;44:107156.

6. Abraham J, Remick JD, Caulfield $\mathrm{T}$ et al. Left ventricular assist device outflow cannula obstruction treated with percutaneous endovascular stenting. Circ Heart Fail 2015;8:229-30.

7. Berg KB, Klodell CT. Outflow graft thrombectomy during HeartMate II left ventricular assist device exchange. Interactive CardioVascular and Thoracic Surgery 2014;19:714-715.Figure Legends:Figure. (A) LVAD parameters derived from device interrogation before and at the time of low-flow alarms, (B) Cardiac CT tomography with 3D reconstruction depicting the site of outflow obstruction (blue arrow), (C) CT angiography 6 months prior without evidence of outflow cannula obstruction, (D) Cardiac CT tomography (coronal axis) suggestive of outflow cannula obstruction. Pathology panels: Cross section of the distal outflow cannula at the bend relief shows a clot consisting of fibrin and blood in the lumen (E) with corresponding representative $\mathrm{H} \& \mathrm{E}$ section $(\mathrm{F})$. Microscopic examination revealed exuberant giant cell reaction between the Dacron and cannula (G). Fibrin platelet material in the lumen (H) does not demonstrate evidence of thrombus organization, confirmed by immunohistochemical stain for vimentin (I). Cross section of the proximal outflow graft at the bend relief shows a clot consisting of fibrin and blood in the lumen $(\mathrm{J})$ and with corresponding representative $\mathrm{H} \& \mathrm{E}$ section $(\mathrm{K})$. Microscopic examination revealed fibrin platelet material between the Dacron and bend relief without evidence of giant cell reaction $(\mathrm{L})$. Clot material in the lumen demonstrated thrombus organization (M) confirmed by immunohistochemical stain for vimentin $(\mathrm{N})$. 


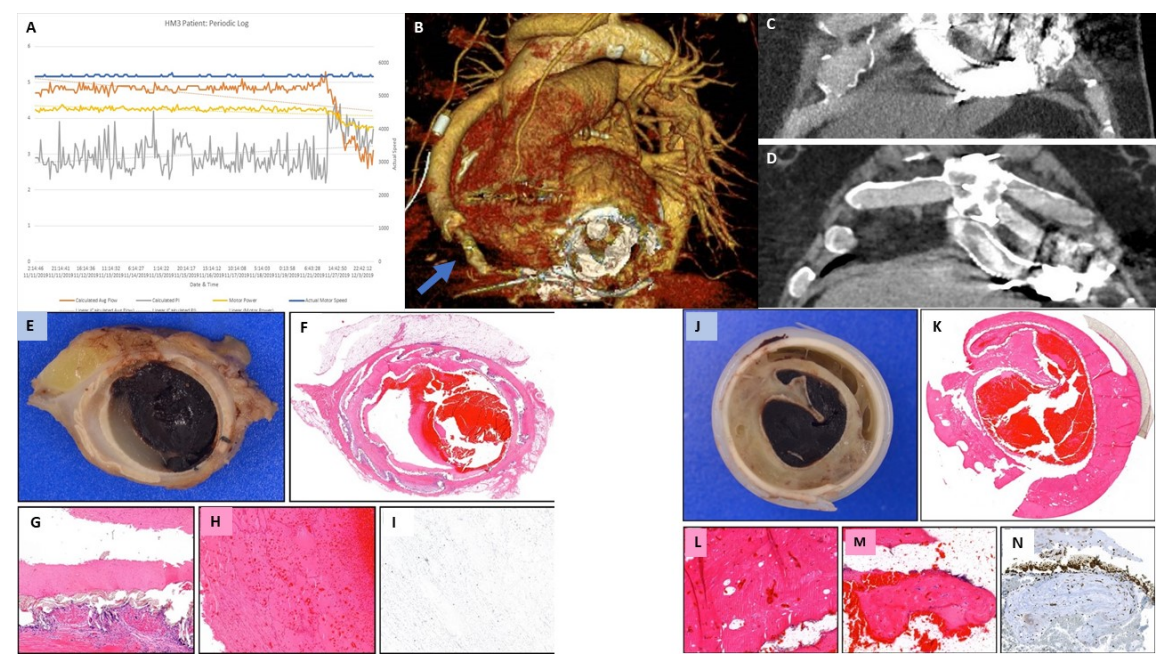

